Regards sur l'économie allemande

Bulletin économique du CIRAC

118-119| 2015

Varia

\title{
Le partenariat contractuel allemand à l'épreuve des corporatismes catégoriels
}

René Lasserre

\section{(2) OpenEdition}

Journals

Édition électronique

URL : http://journals.openedition.org/rea/4888

DOI : $10.4000 /$ rea.4888

ISSN : 1965-0787

Éditeur

CIRAC

Édition imprimée

Date de publication : 31 décembre 2015

Pagination : 15-21

ISSN : 1156-8992

Distribution électronique Cairn

\section{CAIRN INFO}

CHERCHER, REPÉRER, AVANCER.

\section{Référence électronique}

René Lasserre, "Le partenariat contractuel allemand à l'épreuve des corporatismes catégoriels », Regards sur l'économie allemande [En ligne], 118-119 | décembre 2015, mis en ligne le 31 décembre 2017, consulté le 06 mai 2019. URL : http://journals.openedition.org/rea/4888 ; DOI : 10.4000/ rea.4888 


\section{Le partenariat contractuel allemand à l'épreuve des corporatismes catégoriels}

\section{René Lasserre}

L'efficacité du système allemand de régulation sociale et sa capacité éprouvée à opérer un partage socialement équilibré de la valeur ajoutée entre le facteur travail et le facteur capital au sein des différentes branches professionnelles ont, jusqu'à une période récente, reposé sur la stabilité des règles et des pratiques de la négociation collective. Cette dernière est en quelque sorte la charpente à l'abri de laquelle, dans chaque profession, se définissent les enjeux d'un dialogue social structuré qui se prolonge par la concertation entre employeur et salariés dans chaque entreprise.

Pourtant, un certain nombre de conflits récents, répétés et aux effets retentissants, notamment dans le transport aérien et le transport ferroviaire, semblent indiquer que le dialogue social allemand se trouve confronté aux effets déstabilisants, jusqu'alors inédits, de la montée du pluralisme catégoriel. Au point que l'un de ses principes fonctionnels fondamentaux, celui de l'unicité de représentation professionnelle de branche, donne lieu à un débat politique et juridique qui pourrait déboucher, par la voie constitutionnelle, sur une modification de son équilibre d'ensemble.

\section{La genèse historique et l'architecture du système allemand de négociation collective}

La négociation contractuelle est en effet profondément ancrée dans l'histoire sociale allemande : prenant racine dans le contexte de l'essor industriel au tournant des années 1900, elle s'étend pratiquement à tous les secteurs en dehors des forteresses du charbon-acier à la veille de 1914, puis se généralise à partir de 1918 en dépit du contexte économique difficile de la République de Weimar. Vidée de son contenu par la grande dépression, puis euthanasiée dès mai 1933 avec les syndicats par le nazisme, elle renaît de ses cendres avec la réforme monétaire de juin 1948. Celle-ci recrée les bases de l'économie de marché restaurant la libération des prix et la liberté de négocier. La Loi fondamentale de 1949 en consacre le principe, érigeant celle-ci au rang de droit constitutionnel et garantissant aux partenaires sociaux une très large autonomie contractuelle.

Dans ce nouveau contexte de rupture doctrinale avec le dirigisme, où le rôle de l'Etat se voit réduit à la fixation d'un cadre légal minimal, la négociation se modernise et se consolide au début des années 1950 sur une architecture relationnelle que les partenaires sociaux définissent et mettent en place conjointement, tant au niveau global qu'à celui des branches professionnelles.

Tirant les leçons de leurs affrontements passés et confrontées à l'immense défi de la reconstruction, les organisations syndicales et patronales privilégient l'objectif de la cohésion et de l'efficacité, optant de concert et symétriquement pour le principe de l'unité d'organisation et de représentation. Du côté syndical prévaut le principe du syndicat unitaire (Einheitsgewerkschaft) impliquant la neutralité doctrinale, tant politique que religieuse, et sa vocation majoritaire fondée sur la primauté donnée à la défense des intérêts professionnels communs.

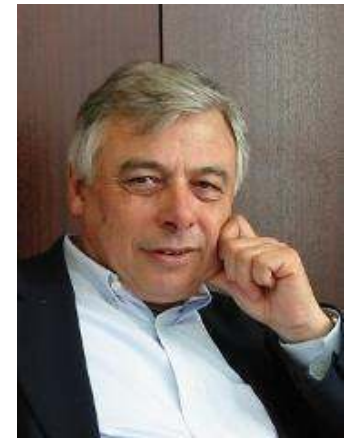

Prof. René Lasserre, Directeur du CIRAC

La renaissance de la liberté de coalition en 1948

Le principe cardinal du syndicat unitaire de branche 
Un pluralisme syndical résiduel

Unité de la représentation socio-professionnelle patronale

Autonomie de négociation et unicité de la représentation contractuelle
De plus, ce principe a été combiné, par souci d'efficacité, au principe uniforme du syndicat d'industrie (Industrieverbandprinzip) qui implique le primat de l'intérêt commun des salariés, lié à leur l'appartenance à une même branche professionnelle, sur les intérêts catégoriels particularistes et souvent contradictoires liés à leur métier. A partir de ce double choix, et notamment de celui de l'abandon définitif du syndicat de métier, le mouvement syndical majoritaire réuni au sein de la grande confédération du DGB s'est organisé prioritairement et uniformément sur la base du syndicat unitaire de branche.

Sans toutefois parvenir à assurer l'unicité totale de la représentation salariée, puisque dans certaines régions industrielles et ouvrières à dominante catholique, notamment en Rhénanie, s'est reconstituée une Confédération des syndicats chrétiens (CGB). Par ailleurs, au plan catégoriel s'est longtemps maintenu dans les secteurs des services marchands (commerce, banque, assurance, services divers...) un syndicalisme d'employés regroupé dans la Deutsche Angestellten-Gewerkschaft (DAG), ayant vocation à faire prévaloir, à côté du syndicat unitaire de branche HBV affilé au DGB, les intérêts spécifiques des employés supérieurs. Dans les branches tertiaires, les deux organisations ont longtemps coexisté, agissant généralement de concert sous la forme d'une communauté tarifaire intersyndicale (Tarifgemeinschaft), jusqu'à ce que la DAG rejoigne finalement Ver.di, le syndicat unifié des services, né en 2001 du regroupement des fédérations du DGB du secteur tertiaire.

Dans la recomposition du mouvement patronal de l'après-guerre, c'est le souci de cohésion et d'efficacité fonctionnelles qui a prévalu, permettant de faire la part entre la défense lobbyiste des intérêts économiques et commerciaux spécifiques, souvent concurrentiels, des fédérations sectorielles d'entrepreneurs, et celle de la représentation des intérêts socio-professionnels communs des employeurs vis-à-vis de leurs interlocuteurs syndicaux ou publics dans des regroupement de branches plus vastes caractérisés par des conditions assez largement homogènes de qualification, de rémunération, et d'emploi de la maind'œuvre. A cette fin, le mouvement patronal a choisi un mode de représentation socio-professionnelle solidaire et unitaire, homothétique à celui du mouvement syndical, dans lequel notamment les employeurs s'efforcent de définir des normes salariales et sociales communes en conciliant, dans le même ensemble professionnel, les intérêts des PME et des grandes entreprises.

Cette architecture symétrique favorise l'institutionnalisation du dialogue social dans les branches qui constituent le niveau privilégié de régulation normative s'appliquant ensuite à une très large majorité des entreprises du secteur. Elle incite les partenaires sociaux à élaborer de part et d'autre des objectifs de négociation homogènes et potentiellement conciliables en fonction de la performance économique de la branche. Sur cette base relativement objective, les deux parties parviennent généralement par la négociation à un partage économiquement et socialement équitable de la valeur ajoutée.

\section{Les principes directeurs du système allemand de négociation}

Depuis la refondation de l'après-guerre, la stabilité du système de négociation salariale et sociale s'est longtemps appuyée, et s'appuie encore très largement, sur trois principes fonctionnels étroitement interdépendants : l'unité organisationnelle symétrique de la représentation syndicale et patronale, le primat donné conjointement par ces dernières à la négociation de branche, et Punicité contractuelle (Tarifeinheit) qui découle de leur très forte représentativité respective. Cette unicité de la régulation se traduit, pour l'ensemble de l'activité économique, y compris dans le secteur public, par la prééminence de conventions collectives unitaires réglant de façon autonome et homogène les différents enjeux socio-économiques de chaque profession : salaires, classifications, conditions d'emploi et de travail. Ce système, fondé sur le principe constitutionnel de l'autonomie contractuelle (Tarifautonomie) des partenaires sociaux - érigée au 
rang de droit social fondamental (Art.9, § 3 de la Loi fondamentale, LF) - garantit ainsi aux acteurs sociaux organisés un très large espace de libre négociation et de régulation, lequel se décline de façon variée et différenciée selon les branches. L'intervention régulatrice uniforme de l'Etat s'en trouve circonscrite à la fixation, par la loi, d'un ordre public social minimal, définissant d'un côté les règles de base de la négociation (Loi sur les conventions collectives), et de l'autre des normes-plancher de protection sociale généralement applicables (législation sur la protection individuelle du travailleur). Ce qui permet tout au plus à la puissance publique, en cas de carence des partenaires sociaux et de vide conventionnel peu fréquents, d'intervenir à la marge et de façon subsidiaire, pour rendre applicables, par simple procédure d'extension, les normes conventionnelles déjà existantes dans un champ professionnel voisin.

Globalement, le système allemand de négociation autonome a fait ses preuves et présente de très solides avantages, qui en font un système de référence internationalement reconnu.

II permet en effet une négociation régulière et récurrente, par laquelle les partenaires sociaux assurent, en toute autonomie, une régulation de tous les enjeux significatifs du partage de la valeur ajoutée dans la quasi-totalité des secteurs d'activité. Et ce, de façon différenciée et flexible selon les données économiques et sociales spécifiques et les performances respectives de chaque profession. Au total, ce système de régulation se caractérise par un degré très faible de conflictualité et une stabilité intra-sectorielle qui assure aux entreprises, qu'elles soient petites et moyennes ou de taille internationale, à la fois prévisibilité et continuité de leurs stratégies.

Pièce maîtresse de l'œuvre de reconstruction, le système de négociation n'a cessé d'enraciner sa légitimité et de démontrer sa capacité alternative à produire puis à garantir du progrès social aussi bien dans les phases de croissance que pendant les périodes de crise. C'est d'ailleurs dans les périodes difficiles qu'il s'est montré le plus efficace, en particulier depuis la fin des années 1990 dans le contexte de la globalisation et celui de la crise de 2008. II a démontré sa capacité à adapter, dans un dialogue social concerté, les conditions de rémunération, d'emploi et de travail dans les grands secteurs de l'économie, et en particulier dans ceux qui sont les plus exposés aux changements structurels liés à la compétition internationale.

En cela, il est considéré comme un des principaux vecteurs institutionnels de la compétitivité allemande, indépendamment des « dividendes sociaux que constitue la garantie d'un niveau élevé d'emploi, de rémunération et d'intégration sociale pour une très large part de la population active.

Ce système de partenariat contractuel, qui a longtemps été le lot commun de l'ensemble de l'économie allemande, se trouve cependant, depuis le tournant des années 2000, progressivement battu en brèche, principalement dans le secteur des services d'intérêt général. Désignés sous le terme générique de Daseinsvorsorge, ils recouvrent les anciens services publics assurés à l'échelon fédéral (grands réseaux d'infrastructure nationaux), régional et local (énergie, transport, hôpitaux, assainissement...).

Ces derniers ont fait en Allemagne l'objet d'une politique systématique de libéralisation des marchés, en application des directives européennes des années 1990. Bien que situés à la périphérie du secteur industriel et commercial, ils n'en revêtent pas moins une importance névralgique pour le fonctionnement de l'économie, puisqu'ils recouvrent les grandes infrastructures de réseaux (électricité, télécommunications, transport ferroviaire et urbain, transport aérien, secteur postal et logistique). Sans parler du secteur hospitalier et d'un ensemble très diversifié d'activités de services aux entreprises et à la personne dans lesquels les structures de qualification et de représentation professionnelles se caractérisent par de fortes disparités catégorielles et statutaires.

\section{Un système}

de régulation sociale structurant, performant et légitime...

... mais potentiellement déstabilisé par les effets de la dérégulation des services d'intérêt général 
Dans ce vaste secteur anciennement de droit public, la régulation sociale contractuelle ne se différenciait pas fondamentalement dans son architecture de celle du secteur privé, puisqu'elle mettait en jeu, par grand secteur d'activité, du côté " patronal », un employeur ou une communauté d'employeurs compétents et, du côté syndical, la fédération syndicale unitaire compétente (ÖTV pour les employés des services publics, ceux de l'énergie, des transports ferroviaires et de la poste). La privatisation et la dérégulation ont totalement changé la donne tant du côté des employeurs que du côté des salariés, de nouveaux acteurs ont émergé pour faire valoir les intérêts spécifiques de certaines catégories dans un marché désormais concurrentiel.

Dans ce nouvel ensemble aux contours et aux structures sectoriels devenus instables, les syndicats de branche, loin d'avoir réussi à consolider leurs positions, parviennent de plus en plus difficilement à assurer la représentation exclusive, voire majoritaire de l'intérêt du plus grand nombre. Dans un certain nombre de secteurs ou de professions importants, comme le trafic aérien, mais où la dynamique de marché et la concurrence internationale rendent le maintien des acquis difficile, les syndicats de branche se heurtent à la montée des corporatismes et se trouvent confrontés à la concurrence de syndicats de métiers très fortement implantés auprès de personnels hautement qualifiés. Ces derniers n'hésitent pas à user de l'influence ou du pouvoir de nuisance qu'ils détiennent par leur position pour faire prévaloir par la grève et par voie d'accords séparés des avantages catégoriels exorbitants.

\section{Emergence des organisations de défense catégorielle}

Ce fut effectivement le cas du transport aérien, où le syndicat CoCkpit (VC), qui représente quelque $80 \%$ des pilotes exerçant dans la compagnie Lufthansa, et $55 \%$ dans près d'une vingtaine de compagnies opérant sur le sol allemand, revendique la représentation exclusive des pilotes. VC a imposé en 2001, sous la menace de grèves successives, une convention collective séparée pour les pilotes qui prévoyait au départ une hausse de rémunérations de $28 \%$ régulièrement renouvelée depuis lors pour des montants plus modestes, non sans recours récurrents à la grève. A côté de VC, le transport aérien compte deux autres syndicats catégoriels très actifs, celui du personnel naviguant (UFO) et celui de la sécurité aérienne (GdF) qui sont en concurrence avec Ver.di-Transport.

Dans le secteur ferroviaire, c'est le Syndicat des Conducteurs de Locomotives (GDL) qui s'est imposé comme syndicat catégoriel le plus influent par l'audience très largement majoritaire $(80 \%)$ dont il jouit auprès des conducteurs de trains de la Deutsche Bahn, le syndicat majoritaire pour l'ensemble de la profession ferroviaire restant le Syndicat du Transport ferroviaire (EVG) affilié au DGB. Depuis 2008, GDL a imposé un accord catégoriel spécifique pour les conducteurs de trains, transformé en 2011, après plusieurs mouvements de grève, en accord-cadre fédéral pour les secteurs voyageurs et marchandises, assorti de primes et indemnités avantageuses. La tentative engagée en 2014 pour étendre cet accord au secteur ferroviaire privé, notamment au niveau régional, n'a pour l'instant pas abouti, en dépit d'une vague de grèves successives

Dans le secteur sanitaire, le Marburger Bund (MB), qui défend les intérêts professionnels des médecins fonctionnaires des hôpitaux communaux et des cliniques universitaires, s'est imposé comme le fer de lance de la représentation catégorielle du personnel médical de haut niveau dans le secteur hospitalier, à côté du syndicat Ver.di des professions médicales et personnels soignants. Le MB a longtemps coopéré avec Ver.di avant de faire cavalier seul en 2005. Ceci en vue d'assurer une représentation autonome des médecins et de faire valoir en 2006, à la faveur d'une grève des médecins hospitaliers, une grille de rémunération spécifique au sein d'un accord séparé pour les médecins dans le cadre de la Convention tarifaire du Service Public (TVöD), régulièrement renouvelé depuis lors.

D'autres organisations catégorielles se sont développées dans la période récente dans des secteurs des services aux entreprises, mal couverts par les syndicats, notamment dans les fonctions de sécurité incendie et de maintenance, et ce à proximité d'infrastructures publiques ou de zones d'activité industrielle. Ces exemples révélateurs ne sont que les plus significatifs parmi la multiplication des accords catégoriels ponctuels, lesquels, pour être fréquents dans le secteur des services d'intérêt général (SIG), touchent aussi d'autres activités des services marchands classiques et des services aux entreprises, mais dont bon nombre échappent à toute régulation contractuelle organisée.

Ces atteintes à la règle coutumière de l'unicité contractuelle traditionnellement assumée conjointement par les partenaires sociaux se sont multipliées depuis 
le tournant des années 2000 et ont affecté chroniquement plusieurs secteurs importants des services d'intérêt général. Elles ont souvent trouvé leurs origines dans des constellations d'intérêts particulières à la sphère publique et donné lieu à des conflits d'ampleur et d'intensité inédites.

Par contraste, il convient de souligner que le bloc central de l'économie allemande de l'industrie de transformation et des grands services concurrentiels du secteur commercial et financier est resté très largement préservé, à la fois du fait de la proximité de la pression du marché et d'une gestion des ressources humaines performante qui s'appuie sur des grilles de qualification homogènes et privilégie la valorisation des compétences et la motivation des personnels. Les tensions propres aux SIG sont donc assez circonscrites, et les risques de contagion au secteur mercantile semblent assez réduits.

\section{Vers une refondation juridique du partenariat contractuel allemand?}

Sans qu'il soit possible d'en dresser un relevé statistique précis, la multiplication des accords catégoriels au cours des dix dernières a été suffisamment significative à la fois dans son intensité et dans ses enjeux pour provoquer un débat public de grande ampleur. Celui-ci a été relayé par un débat juridique de fond, lancé à l'initiative du Tribunal fédéral du Travail lui-même, qui a décidé de modifier sa jurisprudence et, par son arrêt du 17 juillet 2010, de remettre en question le principe même de l'unicité tarifaire.

Le Bundesarbeitsgericht (BAG) a rappelé que le principe de l'unité contractuelle n'avait aucune base juridique légale et ne relevait que d'un droit d'usage découlant des conditions dans lesquelles l'organisation de la négociation collective avait été mise en place et pratiquée par les partenaires sociaux depuis la dernière guerre. En vertu de ce principe ne pouvaient jusqu'alors être reconnus comme légitimes et applicables pour une branche donnée que les accords signés par les syndicats majoritaires, tandis que les accords obtenus par les organisations minoritaires se voyaient dénier toute validité. Le tribunal a considéré que l'application de l'unicité contractuelle avait ainsi pour conséquence de priver arbitrairement les adhérents des organisations minoritaires des garanties contractuelles ainsi obtenues par l'exercice du droit intangible de la liberté de coalition, garanti à l'Art.9, §3 de la Loi fondamentale. Constatant en outre qu'aucune disposition réglementaire n'empêchait l'existence de plusieurs accords dans une même branche, et que par ailleurs, l'expérience en vigueur dans différents secteurs montrait qu'elle pouvait être effectivement praticable, le Tribunal fédéral du Travail a formellement appelé le législateur à intervenir pour rendre possible et organiser les conditions d'une pluralité conventionnelle effective.

Le jugement du BAG, perçu initialement comme un pavé jeté dans la mare et destiné à faire bouger les lignes du dialogue social, a plutôt incité dans un premier temps les organisations professionnelles établies à faire front commun pour préserver leur prééminence régulatrice. Dans une déclaration commune, parue avant même la publication officielle du jugement, les deux confédérations centrales BDA (employeurs) et DGB (syndicats unitaires) ont proposé des aménagements réglementaires permettant de ménager la possibilité d'une négociation minoritaire supplétive. Sans toutefois réellement parvenir à convaincre que ceux-ci soient effectivement praticables.

De leur côté, les syndicats catégoriels les plus offensifs, notamment ceux du trafic aérien et GDL dans le trafic ferroviaire, ont perçu l'arrêt de la juridiction fédérale d'Erfurt comme un encouragement à poursuivre leur offensive en faveur de la concurrence tarifaire. Soucieux de mettre à profit ces nouvelles perspectives et de démontrer leur capacité à imposer leurs revendications spécifiques, ils ont multiplié les campagnes de grèves à répétition au cours des dernières années, causant de graves et coûteuses perturbations dans leurs branches
Remise en question du principe de l'unité tarifaire

Le jugement du Tribunal du Travail du 17 juillet 2010

Contre-propositions du patronat et du DGB

Contre-offensive des syndicats catégoriels 
Législateur : réaffirmation transitoire de l'unité tarifaire

Dans l'attente d'un jugement de Karlsruhe respectives et dans l'activité économique d'ensemble, ce qui n'a pas manqué de susciter un vif mécontentement dans les milieux professionnels et l'opinion.

Devant le durcissement des fronts et sous la pression conjointe des confédérations patronales et syndicales, le gouvernement de Grande coalition a finalement répondu en octobre 2014 à l'injonction de légiférer en présentant un projet de loi optant clairement pour la préservation du principe de l'unité. La Loi sur l'unité tarifaire (Tarifeinheitsgesetz) finalement promulguée le 10 juillet 2015, après un long débat et une audition publique d'experts devant la commission sociale du Bundestag, n'exclut pas la possibilité que puissent être conclues plusieurs conventions dans un espace contractuel donné, mais dispose que dans ce cas de figure, seule doit prévaloir et s'appliquer la convention signée par l'organisation majoritaire. Ce texte de compromis est cependant loin de faire l'unanimité, car il ne répond que formellement au principe d'égalité dans l'exercice du droit de coalition. Pour faire valoir leurs intérêts, les syndicats catégoriels n'ont en effet, dans ce cas de figure, d'autre possibilité que de s'en remettre au bon vouloir des syndicats majoritaires. Dès avant même l'adoption de la loi, il est apparu qu'il reviendra au Tribunal constitutionnel fédéral de Karlsruhe de trancher sur le fond. Celui-ci a entre-temps confirmé qu'il statuerait d'ici la fin de l'année 2016.

Dans cette perspective, les recours en référé que trois syndicats autonomes ont déposé à Karlsruhe au lendemain même de l'adoption de la loi pour demander la suspension immédiate de son application afin que puisse être préservée leur capacité à agir dans les conflits en cours n'avaient qu'une portée conservatoire. Ils n'ont pas vraiment contribué à faire avancer la réflexion sur le fond. Le rejet bienveillant qui leur a été opposé en octobre 2015 semble toutefois indiquer, dans ses attendus, que la Cour n'exclut pas de clarifier sur le fond les conditions par lesquelles les conflits de souveraineté contractuelle pourraient être identifiés et surmontés par voie de conciliation pour satisfaire au principe de l'unité tarifaire.

MAIS CE N'EST LÀ QU'UNE CONSIDÉRATION PROCÉDURALE qui laisse pour l'instant de côté la question, à notre sens essentielle, des modalités de concertation et de coopération à mettre en œuvre entre organisations syndicales d'audiences professionnelles différentes afin qu'elles puissent surmonter la diversité des intérêts catégoriels et parvenir conjointement à la conclusion d'accords majoritaires au sein d'une même branche ou d'une même entreprise. Sur ce plan, les syndicats unitaires de l'industrie allemande ou des grandes branches des services marchands disposent d'une longue expérience pour assurer, soit dans leurs commissions tarifaires internes, soit dans des communautés tarifaires ( Tarifgemeinschaften) intersyndicales, la prise en compte de la diversité des intérêts de leur mandants et les intégrer dans des conventions performantes et légitimes. Les Juges de Karlsruhe devraient pouvoir inviter les parties à définir pour ce faire des procédures de médiation adéquates.

Peut-être est-il aussi quelque peu illusoire de vouloir traiter, selon des principes généraux uniformément applicables pour le cœur de l'économie concurrentielle, des problèmes de régulation socio-professionnelle qui touchent plus particulièrement quelques segments des services d'intérêt général, dont les structures de qualification et les modes de gestion des personnels relèvent de logiques différentes. Plutôt que de s'évertuer à définir un ensemble de normes de portée générale qui risquent de rester sans effet, les Juges de Karlsruhe ne seraient-ils pas mieux inspirés d'édicter quelques règles applicables au seul secteur de la Daseinsvorsorge? Le partenariat social allemand sera d'autant mieux préservé dans les principes qui en fondent l'efficacité générale qu'il pourra s'appuyer, dans ce secteur particulier, sur des procédures adaptées. 


\section{Indications bibliographiques}

-BDA-DGB, Funktionsfähigkeit der Autonomie sichern - Tarifeinheit regeln. Gemeinsame Erklärung, 02-06-2010

-BOURGEOIS I., «Travail : fin de la convention unique de branche », in Regards sur l'économie allemande, $\mathrm{n}^{\circ} 97 / 2010$

- BUNDESARBEITSGERICHT, Urteil vom 7.7.2010, Grundsatz der Tarifeinheit - Rechtsprechungsänderung, 4 AZR 549/08

-DAUBLER W., « Empörung gegen BAG - Urteil ungerechtfertigt », Junge Welt, 25-06-2010

- Deutscher Bundestag, Ausschuss für ARBeit und Soziales, Kontroverse Expertisen zum Tarifeinheitsgesetz, 04-05-2015; www.bundestag.de/dokumente/textarchiv/2015:kw19 (texte et vidéo)

- Hazouard S., Lasserre R., UterWedde H. (ed), Relations sociales dans les services d'intérêt général. Une comparaison France-Allemagne, Ed. du CIRAC, Cergy-Pontoise, 2011

Voir en particulier :

BRANDT T., SChULTEN T., "Le rôle de la libéralisation et de la privatisation des services publics dans l'érosion de la convention collective de branche en Allemagne "

GreEF S., KALASS V., "Les relations sociales à la Deutsche Bahn : nouveaux acteurs et évolution des rapports de force sur fond de privatisation et de libéralisation "

Schroeder W., Greef S., "Nouveaux acteurs de la représentation des intérêts des salariés en Allemagne 》

- HUNDT D., «Aufhebung der Tarifeinheit. Welche Folgen hat das Nebeneinander mehrerer Tarifverträge in einem Unternehmen ? », Ifo Schnelldienst, 9/2012

- IG MetalL, Gesetz zur Tarifeinheit : Hintergrund, Ziele, Inhalte, Bewertung, 21-04-2015

- LESCH H., "Tarifpluralität und Lohnbildung », Ifo Schnelldienst, 9/2012

- LESCH H., « Spartengewerkschaften - Droht eine Destabilisierung des Flächentarifvertrages ? », in Sozialer Fortschritt 57/6, 2008

- SACHVERSTÄNDIGENRAT ZUR BEgUTACHTUNG DER GESAMTWIRTSCHAFTLICHEN ENTWICKLUNG, "Das Ende der Tarifeinheit : kein gesetzgeberischer Aktionismus ", extrait de Jahresgutachten 2010/11

-Ver.di Bundesvortand, Stellungnahme zum Entwurf eines Gesetzes zur Tarifeinheit, 18-112014. 